\title{
Unimanual and bimanual weight perception of virtual objects with a new multi-finger haptic interface
}

\author{
Christos D. Giachritsis*, Manuel Ferre, Jordi Barrio, Alan M. Wing \\ University of Birmingham, School of Psychology, Edgbaston, Bimingham, West Midlands B15 2TT, United Kingdom
}

\section{A R T I C L E I N F O}

\section{Keywords:}

Haptics

Weight perception

Haptic interfaces

Psychophysics

\begin{abstract}
A B S T R A C T
Accurate weight perception is important particularly in tasks where the user has to apply vertical forces to ensure safe landing of a fragile object or precise penetration of a surface with a probe. Moreover, depending on physical properties of objects such as weight and size we may switch between unimanual and bimanual manipulation during a task. Research has shown that bimanual manipulation of real objects results in a misperception of their weight: they tend to feel lighter than similarly heavy objects which are handled with one hand only [8]. Effective simulation of bimanual manipulation with desktop haptic interfaces should be able to replicate this effect of bimanual manipulation on weight perception. Here, we present the MasterFinger-2, a new multi-finger haptic interface allowing bimanual manipulation of virtual objects with precision grip and we conduct weight discrimination experiments to evaluate its capacity to simulate unimanual and bimanual weight. We found that the bimanual 'lighter' bias is also observed with the MasterFinger-2 but the sensitivity to changes of virtual weights deteriorated.
\end{abstract}

\section{Introduction}

Effective and safe manipulation of objects requires accurate perception of physical properties such as size, shape and weight. For example, weight will inform us about how heavy an object is so that we can adjust our posture and grip force to achieve effective manipulation. When an object feels heavy, we may chose to use both hands to lift it and/or increase our grip force to maintain a secure grip. In addition, weight perception may affect the way we carry out precision tasks which require deployment of exact forces by the user at the moment of contact between the effectors (e.g., surgical probe) and the effected objects (e.g., human organ). Research on haptics suggests that people perceive weight using both cutaneous and proprioceptive cues such as skin pressure, tactile flow and muscular activity [10,1]. For example, when we lift objects with rougher surfaces or shape facilitating effortless grip then we perceive them as lighter than objects with smoother surfaces or shape resulting in arduous grip. Both smooth and 'arduous grip' shapes demand the application of greater grip forces in order to lift and/or hold the object. This has been shown to result in a greater 'sense of effort' and heavier weight percept [2-4]. Further studies have shown that the state of the muscles (e.g., paresis, anaesthesia) or the level of muscular activity (e.g., fatigue, changes in arm weight, and posture) may also affect 'sense of effort' and weight

\footnotetext{
* Corresponding author. Tel.: +44 121 4277040; fax: +44 1214144897.

E-mail address: c.giachritsis@bham.ac.uk (C.D. Giachritsis).
}

perception $[5,6,12,14]$. Therefore, loss, impairment or restriction of cutaneous and/or proprioceptive feedback seems to affect our ability to accurately perceive the weight of an object.

Haptic interfaces are widely used to simulate physical objects for different type of applications including micro-surgery and assembly. Most common haptic interfaces (e.g., PHANToM, Delta) are based on one-point-contact force-feedback using a thimble or probe. This type of interfaces, primarily engage the proprioceptive system at different joints (e.g., wrist, elbow, and shoulder) depending on the size of their working space and may provide (depending on their specifications) realistic geometrical representations of simulated objects through haptic exploration. Nonetheless, the use of thimble results in a constant pressure on the skin at the fingertips and, thus, providing unreliable cutaneous information about the physical properties of the virtual objects, including weight. As a consequence, virtual weight may be misperceived. Furthermore, it may be difficult to eliminate the weight of the device which could result in further misperception of weight. Combining more than one device in order to enable multipoint-contact for unimanual and/or bimanual manipulation could easily multiply this problem. For example, the forces applied by the different devices should be spatio-temporarily matched. In the case of bimanual manipulation, the system should also be able to compensate for the different positions and force exerted by the two hands.

Here, we present a weight discrimination study which evaluates unimanual and bimanual weight perception in a desktop environment using a new force-feedback haptic interface which enables multi-point contact manipulation of virtual objects, the 


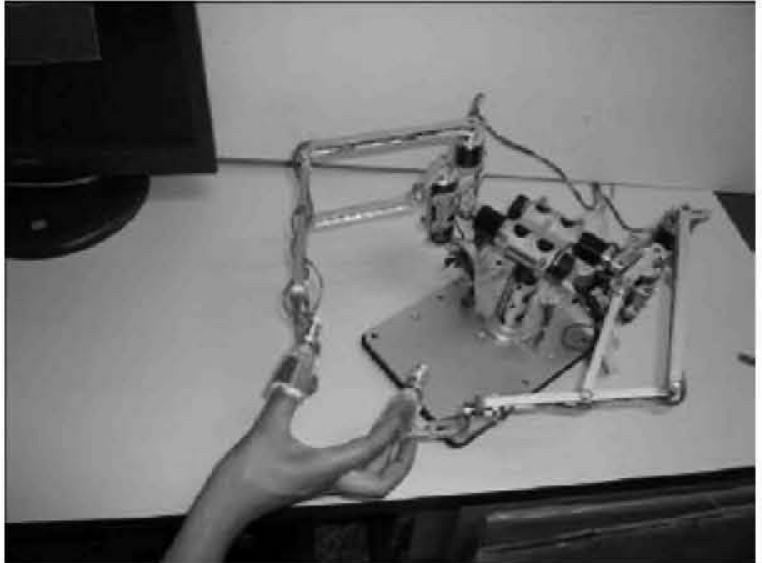

Fig. 1. MasterFinger-2. The thumb and the index finger are inserted in their corresponding thimbles. The thimbles are linked to the common base of MF- 2 via 6 DoF and 3 motors per finger that allows reflecting forces in any direction.

MaterFinger-2 (MF-2) [11]. The main advantage of MF-2 over existing one-point contact force feedback devices is that allows precision grip with large workspace relative to the physical size of the device. For example, when coupling two PHANToMs 1.5 to allow precision grip manipulation the workspace reduces from $270 \mathrm{~mm}^{3}$ to $100-150 \mathrm{~mm}^{3}$. The new MF-2 includes a redundant DOF allowing a larger ellipsoid workspace of $400 \mathrm{~mm} \times 400 \mathrm{~mm} \times 200 \mathrm{~mm}$ (XYZ) to be created and used. An additional advantage is that it is built as open architecture permitting the incorporation of other devices such as sensors and actuators. The present study required the participants to judge virtual weights lifted with the right hand and two hands and then compared the data to those obtained from a previous study which used real weights (part of these data were reported in [8]).

\section{Materials and methods}

\subsection{Apparatus}

In order to generate virtual weights we used the MF-2 device, which is based on a robot-arm design with two arms and allows the user to use precision grip to manipulate virtual objects. Each arm has a serial-parallel structure with 6DOF for movement and 3DOF for force reflection allowing grasp movements in any direction. The arms are connected to the base of the device through an additional actuator to increase workspace and introduce an additional DOF. This 'redundant' DOF is used to monitor the path of movement of the fingers and intervene to avoid collision (Fig. 1). This configuration allows a spherical workspace without collisions between finger links.

The forces exerted by the user where registered by three Flexiforce sensors which were inserted in each thimble (Fig. 2). These sensors were used to measure normal and tangential forces exerted by each finger. The first sensor (Sensor 1) was placed at the bottom of the thimble in direct contact with the fingertip and measured normal force. The other two sensors (Sensors 2 and 3 ) were placed at the lateral sides of the thimble and their output was used to compute the tangential forces. Thus, Sensor 1 measured grip forces and Sensors 2 and 3 measured load forces. In order to increase reliability of force readings, the Flexiforce sensors were placed between two aluminium plates with same shape and size. Tests that conducted to check the reliability of the readings during grasping tasks showed a maximum error of $15 \%$ which was considered to be acceptable since the time evolution of forces promptly corresponds to the forces applied in real grasping tasks.

In order to enable bimanual manipulation of virtual objects, two MF-2 devices were coupled. They were placed upside-down on an aluminium frame in order to remove the bases of the two devices from the desktop to allow the two hands to move freely across the workspace. The user was provided with virtual visual feedback about the position of the hands and the object based on the position and orientation of the index and thumb thimbles (Fig. 3). Feedback forces were calculated on the basis of the location of the thimbles and were proportional to the finger-object penet ration. The forces were delivered to the user when a collision between a finger and the object was detected. In the present set up the force ware contained in a horizontal plane.

During unimanual manipulation, the load of the object was simulated by applying equal vertical forces to both thimbles simultaneously (i.e., $50 \%$ of the total load forces are applied to the index and $50 \%$ to the thumb). During bimanual manipu-

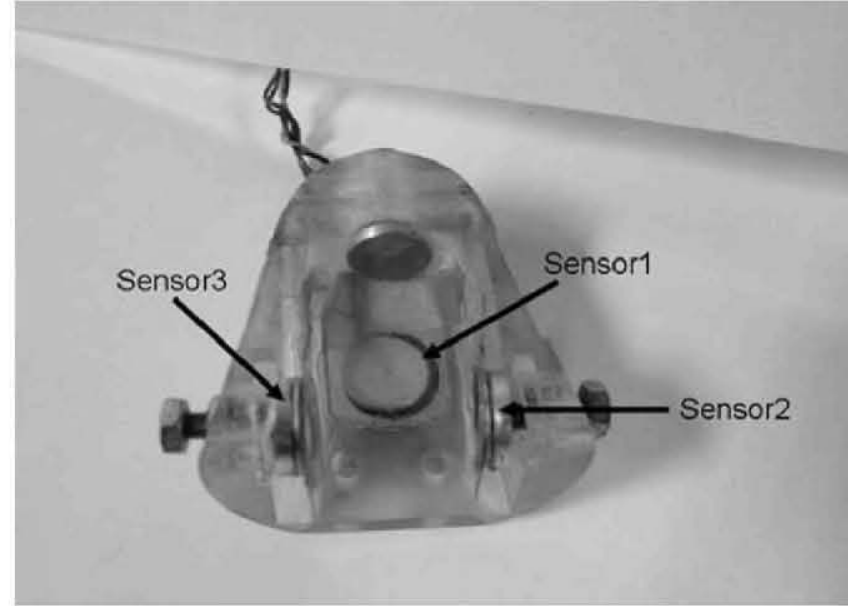

Fig. 2. Thimble developed for measuring the forces applied by the user during the grasping task. Normal forces are mainly detected by Sensor 1 and tangential forces by Sensor 2 or Sensor 3.

lation, the load forces were distributed equally among all four fingers (i.e., 25\% per finger).

\subsection{Stimulus}

The minimum virtual weight which could be simulated consistently by MF-2 over the entire workspace was $75 \mathrm{~g}$. The MF- 2 was capable of simulating forces with smaller magnitude but due to mechanical restrictions the simulation of smalle than $75 \mathrm{~g}$ forces was not consistent across the entire workspace. In the present experimental set up, during the vertical weight lifting, participants approached the maximum $Z$ value of $200 \mathrm{~mm}$. Thus we selected $75 \mathrm{~g}$ as the minimum simu lated weight and used it as the step size (i.e., the minimum difference between two weights) and the minimum weight of a range of seven weights: $75,150,225,300$ (the standard weight), 375,450 and $525 \mathrm{~g}$. The virtual weights were generated by the MF-2 and presented as visuo-haptic boxes with constant size of $100 \times 170 \times 150 \mathrm{~mm}$ $(W \times D \times H)$. The width of the precision grip was determined by the width of the virtual box while the distance between left and right grip in bimanual lifting was determined by its depth. The advantage of the short depth is that it minimises the risk of torques affecting weight judgments in the unimanual lifting by allowing the user to grasp the box near the virtual centre of mass.

\subsection{Procedure}

Eight postgraduate students (average age of 26.1 years old) of the Universidad Politécnica de Madrid volunteered to participate in the study and were all naïve as to the purpose of the experiment. They were all right-handed and they placed their indices and thumbs into the thimbles in order to manipulate the virtual object with a precision gip. Similar experimental procedures and conditions that were used in [8] were also used here; that is, a temporal $2 \mathrm{AFC}$ paradigm and two conditions. The experimental condition (exp), which tested weight discrimination between the right hand (RH) and both hands (BH) and a control condition (ctrl), which tested

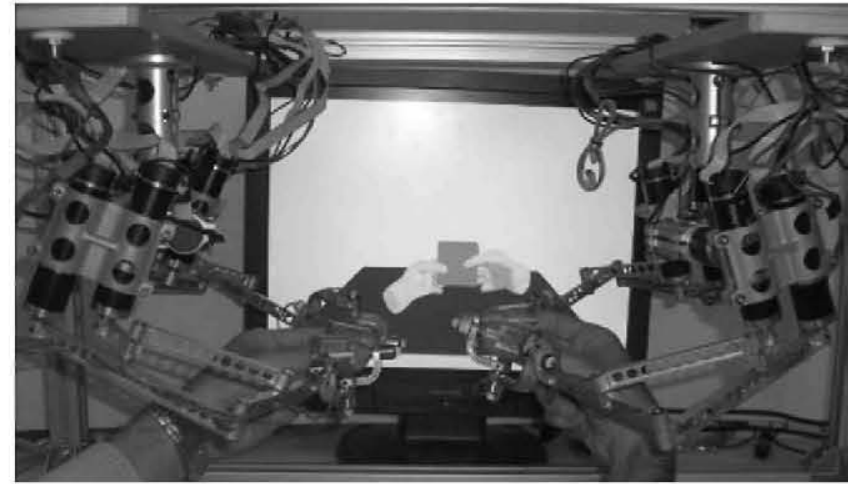

Fig. 3. Experimental setup for unimanual versus bimanual grasping. User lifts a virtual object with one or two hands. A graphical simulation shows evolution of the task. The haptic devices guide the hand postures and reflect forces according to the object manipulation. 
weight discrimination with the right hand only. Each trial consisted of three phases: in the first phase, participants lifted a test/standard box with $\mathrm{RH} / \mathrm{BH}$ (exp) or RH (ctrl); in the second phase, they lifted the standard/test box with $\mathrm{BH} / \mathrm{RH}$ (exp) or $\mathrm{RH}(\mathrm{ctrl})$ and, in the third phase, they reported verbally which box felt heavier. The presentation sequence of standard and test weight was balanced and the trials were randomised in each session. Each test weight was compared twelve times against the standard weight. Therefore, each participant responded 168 times in the experimental condition and 84 times in the control condition. Each session lasted approximately $45 \mathrm{~min}$ including a short break.

\section{Results}

In order to evaluate performance with real and virtual weights, individual data from the control and experimental conditions were used to build a psychometric function. This function would provide the subjective perception of the standard virtual weight or point of subjective equality (PSE) as well as the individual sensitivity to virtual weight changes or discrimination thresholds (DL) [7]. The psignifit [9] software was used to fit individual data from the real and virtual weight experiments with the following commonly used psychometric function, $\psi(x)$,

$\psi(x ; \alpha, \beta, \gamma, \lambda)=\gamma+(1-\gamma-\lambda) F(x ; \alpha, \beta)$,

where $\gamma$ is the lower and $1-\lambda$ the upper bound of the function [13]. The psychometric function is based on the two-parametric logistic rule,

$F(x ; \alpha, \beta)=\frac{1}{1+e^{-(x-\alpha) / \beta}}$,
Virtual Weight $(\mathrm{g})$

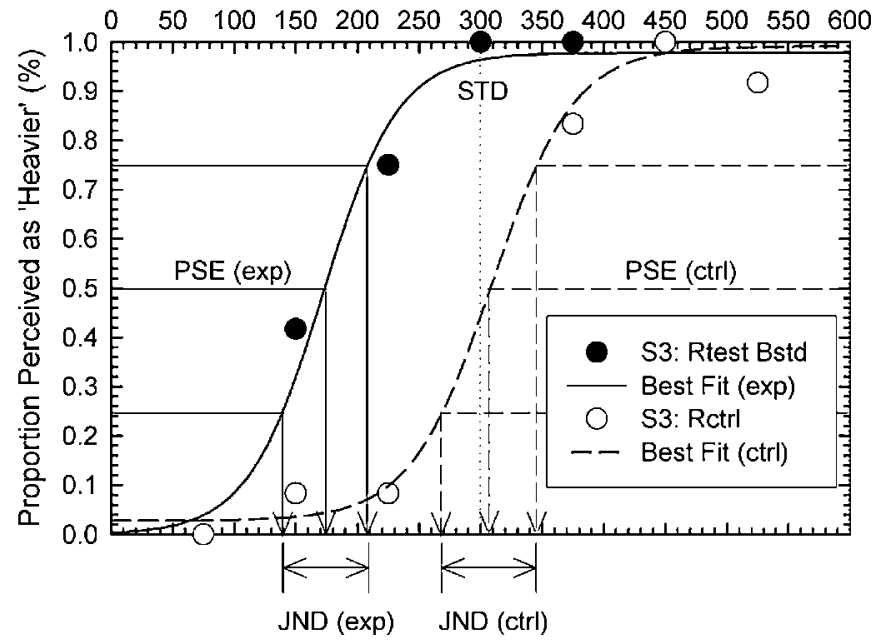

Fig. 4. Example of fitting the psychometric function on data from participant S3. Each data point is based on 12 responses. The overall PSE and JND for the two conditions were obtained and compared (see text for more detail).

where $\alpha$ is location of the function on the $x$-axis and $\beta$ is its slope. The parameter $\alpha$ coincides with the PSE and the parameter $\beta$ indicates how sensitive the users are in detecting changes in virtual weight; that is, the steeper the function the greater the sensitivity. The DL was calculated on the basis of the $25 \%$ and $75 \%$ thresholds

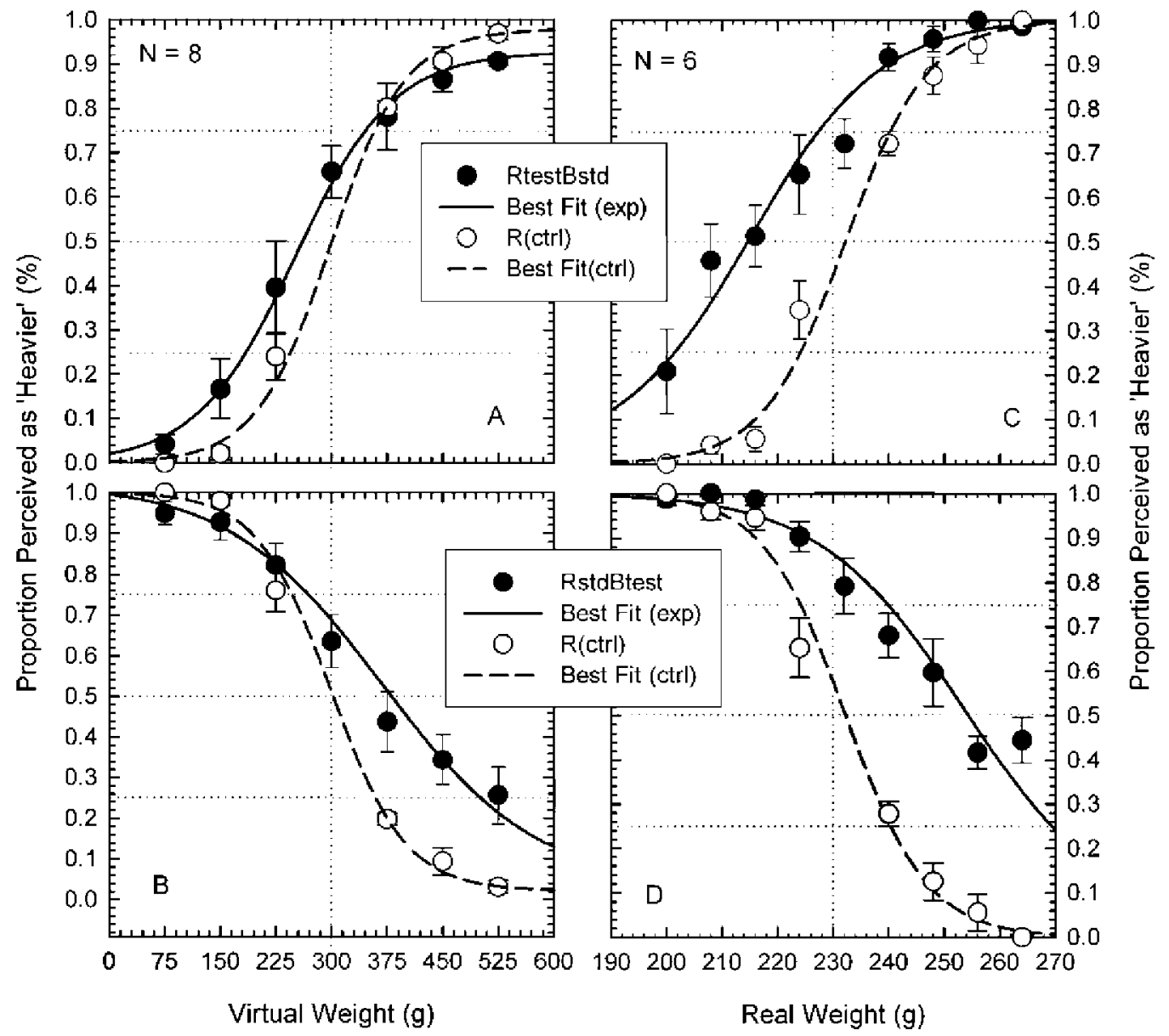

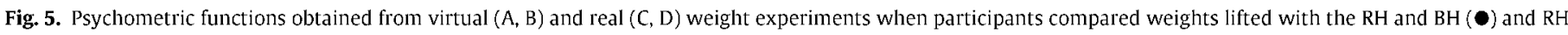
only (O). Error bars represent standard error. 

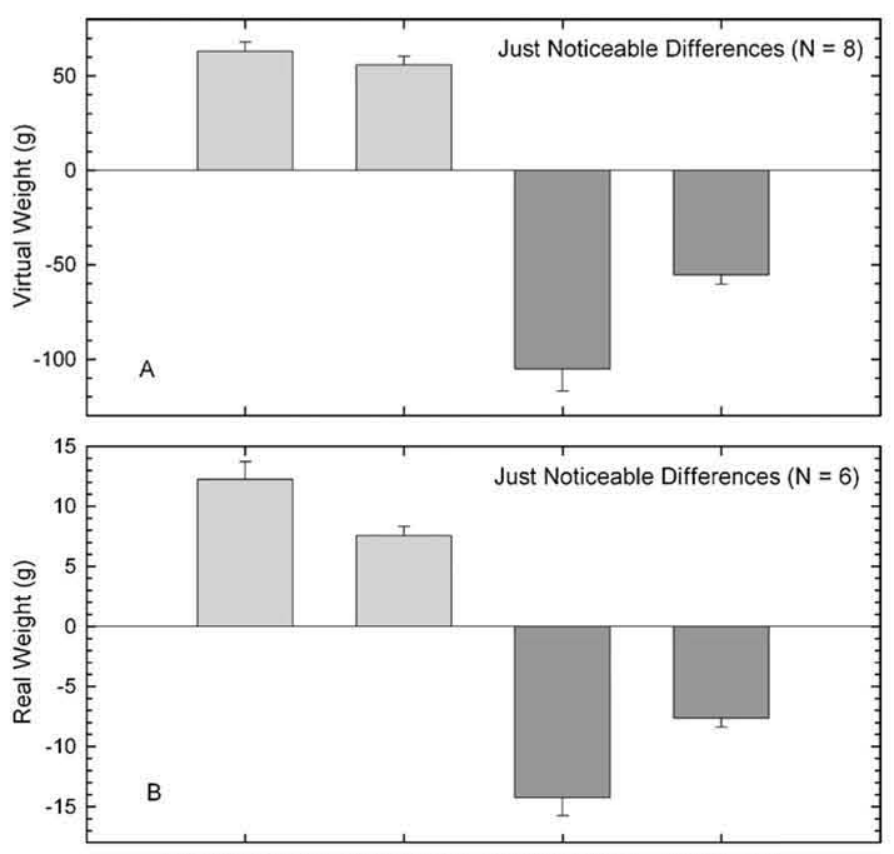

$\mathrm{RH}$ (test)-BH(std) $\mathrm{RH}$ (test_ctrl) $\mathrm{RH}(\mathrm{std})-\mathrm{BH}$ (test) $\mathrm{RH}$ (std_ctrl)

Condition and Presentation Order

Fig. 6. Average just noticeable differences (JND) observed with virtual (A) and real (B) weights. Error bars represent standard error.

as $\left|T_{25}-T_{75}\right| / 2$. An example of fitting the logistic rule to obtain the psychometric function is shown in Fig. 4.

Results showed very similar performances with both virtual and real weights. Bimanual manipulation had a twofold effect on weight perception (Fig. 5). First, bimanually lifted weights felts heavier than weights lifted with the right hand only: the PSE of the experimental condition shifted to the left of the PSE of the control condition. A paired $T$-test showed that this effect was statistically significant with real weights when the standard weight was lifted with either the right hand $\left(T_{(5)}=7.595, p=0.001\right)$ or both hands $\left(T_{(5)}=-4.233, p=0.008\right)$. However, with virtual weights this effect was found to be statistically significant only when the standard weight was lifted with the right hand $\left(T_{(7)}=2.821, p=0.026\right)$. Second, the JND of the experimental condition was greater than the JND of the control condition; that is, sensitivity to changes in virtual weight reduced when users compared weights lifted with the right hand and both hands (Fig. 6). A paired T-test showed that this effect was statistically significant with real weights when the standard weight was presented in the right hand $\left(T_{(5)}=-4.321, p=0.008\right.$ ) and both hands $\left(T_{(5)}=2.62, p=0.047\right)$. Similarly, the effect was statistically significant on JND with virtual weights when the standard weight was presented in the right hand $\left(T_{(7)}=-4.572, p=0.003\right.$ ) and both hands $\left(T_{(7)}=2.732, p=0.029\right)$.

Furthermore, a comparison of the PSE of the control conditions with real and virtual weights (Fig. 7) showed that weight discrimination deteriorated with virtual weights. The JND with real weights was about $8 \mathrm{~g}$ while the JND with virtual weights was about $56 \mathrm{~g}$. Even though the JND with virtual weights was lower than the step size of $75 \mathrm{~g}$, Weber's fraction showed that it was nearly six times higher than the predicted $9.8 \mathrm{~g}$.

\section{Discussion}

The results of the present study have shown that weight discrimination performance with virtual weights generated by MF-2 was similar to real weights. Real weights lifted with the right hand felt heavier than weights lifted with both hands in both presenta-

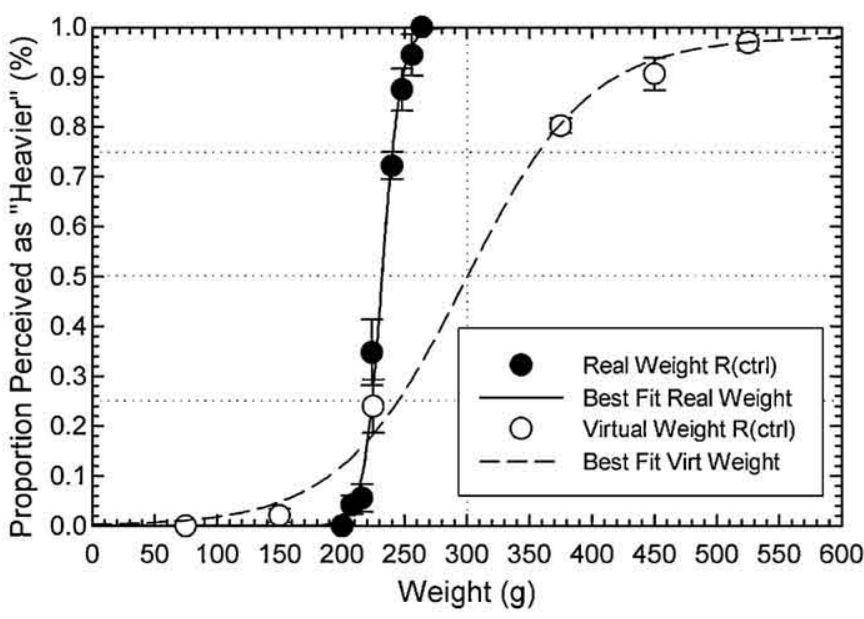

Fig. 7. Comparison of weight discrimination performance with real ( ) and virtual (O) weights. Error bars represent standard errors.

tion orders [8]. The same effect was observed with virtual weights when the standard weight was lifted with the right hand. Moreover, the JND when comparing weights lifted with the right hand and both hands was worse than when comparing weights lifted with the right hand only [8]. This was observed with both real and virtual weights and with both presentation orders. Nonetheless, weight discrimination with virtual weights was worse than with real weights: Weber's fraction obtained from the control condition (i.e., weights lifted with the right hand only) with both real and virtual weights showed that sensitivity to weight changes was reduced nearly six times.

There may be at least two reasons for the deterioration of weight sensitivity with virtual weights. First, participants had no reliable cutaneous information about the weights and therefore relied primarily on proprioceptive feedback to make weight judgments. The absence of cutaneous information could have contributed to the deterioration of weight sensitivity. Research has shown that physical properties of an object such as surface texture, which is perceived through cutaneous feedback, can affect weight judgments [4,3]. Second, during lifting, MF-2 makes small adjustments of the horizontal forces in order to cancel out excessive grip force and maintain a constant size of the virtual box. These small perturbations could have interfered with proprioceptive information from vertical forces simulating virtual weight. If participants were not able to ignore these horizontal perturbations then their ability to accurately perceive virtual weight could have been affected. Research in weight perception using objects with shapes that do not facilitate easy grip may support this suggestion [2]. In these studies, the lack of effective grip may have resulted in constant readjustment of the grip during lift in order to secure the object. These readjustments, which engaged the proprioceptive system, could have caused similar horizontal perturbations with the ones observed in the present study interfering with proprioceptive information about weight.

Despite these limitations, the bimanual MF-2 set up has managed to simulate effectively unimanual and bimanual weight sensation and establish a distinct presence for each of them. Further improvements on the realism of weight perception should be possible if hardware and software design could address the above issues. Haptic interfaces with effective unimanual and bimanual weight simulation could offer professionals the opportunity to train in precision tasks which require accurate weight perception and scientists the possibilities to investigate dynamic aspects of weight perception (e.g., sensitivity to weight changes during object displacement) which is very difficult or even impossible with real weights. 
Finally, the bimanual 'lighter' bias observed with both real and virtual weights sets further questions about the factors that may have produced it. For example, is it based on different peripheral signals or imperfect central integration of weight information from both hands? It could be possible that employment of four digits to lift the same weight may result in more effective grip (e.g., due to elimination of torques which can be present during unimanual lifting) and therefore a weaker 'sense of effort' and 'lighter' weight percept. However, it could also be the case that the central nervous system may receive exactly the same peripheral weight signals but the integration of this information from both hemispheres may be imperfect. In order to answer these questions further studies are necessary.

\section{Conflict of interest}

The authors would like to declare that there are no competing financial interests or other conflict of interest.

\section{Acknowledgments}

This work was funded by the European Commission under the IMMERSENCE Integrated Project of the Sixth Frame Program (IST4-027141-IP).

\section{References}

[1] E.E. Brodie, H.E. Ross, Sensorimotor mechanism in weight discrimination, Percept. Psychophys. 36 (1984) 477-481.
[2] J.R. Flanagan, C.A. Bandomir, Coming to grips with weight perception: effects of grasp configuration on weight heaviness, Percept. Psychophys. $62(6)(2000)$ $1204-1219$.

[3] J.R. Flanagan, A.M. Wing, Effects of surface texture and grip force on the discrimination of hand-held loads, Percept. Psychophys. 59 (1) (1997) 111-118.

[4] J.R. Flanagan, A.M. Wing, S, Allison, A. Spenceley, Effects of surface texture on weight perception when lifting objects with a precision grip, Percept. Psychophys. 57 (1995) 282-290.

[5] S.C. Gandevia, D.I. McCloskey, Effects of related sensory inputs on motor per formances in man studied through changes in perceived heaviness, J. Physiol. 272 (1977) 653-672.

[6] S.C. Gandevia, D.I. Mccloskey, Sensations of heaviness, Brain 100 (1977) 345-354.

[7] G.A. Gescheider, Psychophysics: The Fundamentals, 3rd ed., LEA, New Jersey, 1997.

[8] C.D. Giachritsis, A.M. Wing, Unimanual and bimanual weight discrimination in a desktop setup, in: M. Ferre (Ed.), Proceedings of Eurohaptics '08 (Madrid, June 10-13, 2008), LNCS series, Springer-Verlag, Berlin/Heidelberg, 2008, pp. $378-382$.

[9] http://www.bootstrap-software.org/psignifit

[10] D.I. McCloskey, Muscular and cutaneous mechanisms in the estimation of the weights of grasped objects, Neuropsychologica 12 (1974) 513-520.

[11] M. Monroy, M. Oyarzabal, M. Ferre, A. Campos, J. Barrio, MasterFinger: multifinger haptic interface for collaborative environments, in: M. Ferre (Ed) Proceedings of Eurohaptics '08 (Madrid, June 10-13, 2008), LNCS series, Springer-Verlag, Berlin/Heidelberg, 2008, pp. 411-419.

[12] H.E. Ross, E.E. Brodie, A.J. Benson, Mass-discrimination in weightlessness and readaptation to earth's gravity, Exp. Brain Res. 64 (1986) 358-366.

[13] F.A. Wichmann, N.J. Hill, The psychometric function. I. Fitting, sampling and goodness of fit, Percept. Psychophys. 63 (2001) 1293-1313.

[14] A. Wing, C. Giachritsis, R. Roberts, in: E. Pye (Ed.), Weighing Up the Values of Touch, The Power of Touch, Left Coast Press, Walnut Creek, 2007, pp. 31-44. 